\title{
Interpretation of SAR images in urban areas using simulated optical and radar images
}

\author{
Junyi Tao, Gintautas Palubinskas, Peter Reinartz \\ Remote Sensing Technology Institute \\ German Aerospace Center (DLR) \\ PO Box 1116, 82230 Weßling, Germany \\ Junyi.Tao@dlr.de
}

\author{
Stefan Auer \\ Remote Sensing Technology \\ Technische Universität München \\ Arcisstr.21, 80333, München, Germany \\ Stefan.Auer@bv.tu-muenchen.de
}

\begin{abstract}
Because of the all-weather and all-time data acquisition capability, high resolution space borne synthetic aperture radar (SAR) plays an important role in remote sensing applications like earth mapping. However, the visual interpretation of SAR images is usually difficult, especially for urban areas. This paper shows a method for visual interpreting SAR images by means of optical and SAR images simulated from digital elevation models (DEM), which are derived from LiDAR data. The simulated images are automatically geocoded and enable a direct comparison with the real SAR image. An application for the simulation concept is presented for the city center of Munich where the comparison to the TerraSAR-X data shows good similarity. The simulated optical image can be used for direct and quick identification of objects in the corresponding SAR image. Additionally, simulated SAR image can separate multiple reflections mixed in the real SAR image, thus enabling easier interpretation of an urban scene.
\end{abstract}

\section{INTRODUCTION}

High resolution space borne synthetic aperture radar (SAR) plays an important role for earth mapping and monitoring because of its all-weather and all-time data acquisition capability. For the same reason, SAR images are often the only available data in crisis situations such as earthquakes, tsunamis, flooding etc.

However, SAR images are difficult to be visually interpreted. The speckle phenomenon in SAR images leads to imprecise object borders; distortion effects like shadowing and layover make the analysis of SAR images difficult; multiple scattering leads to bright lines or point signatures and causes high local contrasts in intensity. These effects mostly appear in SAR images of urban areas where man-made objects with different height, shape of roof, building-materials etc. stand close to each other. Nevertheless, these effects are sources of information which are not available in optical images. For instance, bright lines caused by double reflection of radar signal indicate the boundaries of buildings [5]. Point signatures are strong hints for buildings [6] and provide information about facade details such as windows or balconies [7].

For exploiting signatures in SAR images, the interaction of signal with the real world has to be understood. To this end, methods for the interpretation of SAR images in urban areas are needed.
SAR simulators support the interpretation of SAR images. Different concepts for SAR simulation are differentiated and classified in [3]. Another helpful source of information are optical images with special acquisition geometry [1] which are usually difficult to obtain with space borne systems.

In this paper, a method is presented for supporting the visual interpretation of SAR images with simulated optical and radar images using a digital elevation model (DEM) derived from LiDAR data. We use RaySAR [2] for SAR data simulation and the open-source ray tracing software POV-Ray for optical data simulation. By defining appropriate simulation parameters, we obtain SAR and optical images with the same extension and pixel size. They are geocoded and directly comparable to real SAR images.

The paper is structured as follows. In section II the method for processing simulated optical and SAR images and their geocoding is presented. Section III shows the results of the simulated images and the comparison with TerraSAR-X (TSX) images. Finally, the potentials and limitations of the method are discussed in section IV.

\section{PROCESSING OF SIMULATED IMAGES}

For the simulation step, we use a digital elevation model (DEM) based on LiDAR data. The incidence- and azimuth angle of the imaging system is adapted to the real SAR image. In addition, the pixel size of the real SAR image in East and North direction is needed for the geocoding

By presenting the geometrical relationship between SAR images and optical images we give reasons why a simulated optical image with a viewing direction orthogonal to that of a SAR sensor can support the interpretation of a SAR image. Thereafter, the simulation of optical data and SAR data by means of POV-Ray and RaySAR is introduced respectively. Especially, we present the geocoding of the simulated optical and SAR images which enables a direct comparison with the real SAR images.

\section{A. Geometrical relationship between SAR and optical images}

SAR sensors and optical sensors follow very different acquisition principles: a SAR sensor measures the distance between the object and sensor and projects the signal intensity 
on an azimuth-range map; an optical camera measures the angle of the object in its viewing direction and projects the signal intensity on a sensor (e.g. CCD) with perspective projection.

SAR images and optical images are usually very different in their geometric behavior, which result in different positions of objects even after orthorectification. The reason is that the images are normally orthorectified using a DEM with low accuracy (e.g. derived from the Shuttle Radar Topography Mission (SRTM)) in which high buildings are not represented. However, in some special acquisition geometry, the SAR and optical images are very similar. In [1] an optical image of WorldView-1 and a SAR image of TSX are shown with a similar displacement of objects. The prerequisite for that is that the two sensors fly in the same direction and the sum of the incidence angles should be approximately 90 degree.

But there are two main problems for the interpretation of SAR images with optical images: (1) the prerequisite is too restricted, because of the fixed azimuth and incidence angle, also the condition of weather and time. (2) Because of the perspective projection of the optical sensor data, the displacements of objects in the two images should theoretically only be the same in one line.

Based on this idea, we propose to provide a simulated optical image based on an orthographic projection instead of perspective projection. Figure 1 . shows the principal of the optical simulation. If the viewing directions of the virtual camera and the SAR sensor are orthogonal, the displacement of objects will be the same.

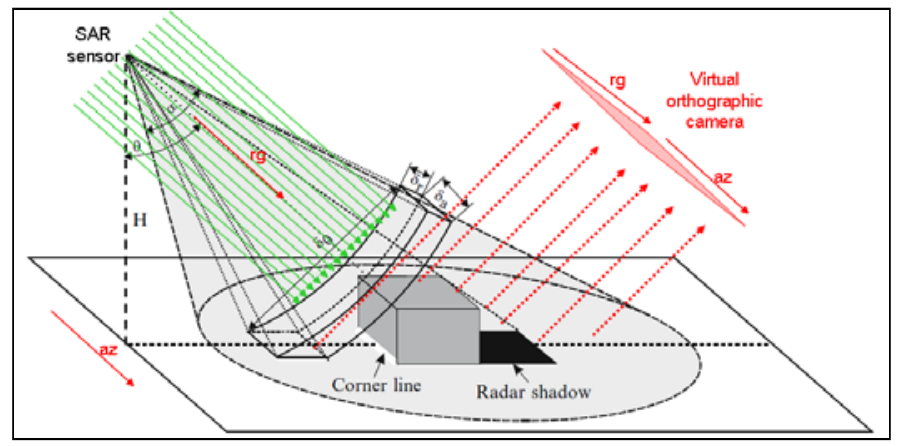

Figure 1. Principal of optical simulation

\section{B. Optical simulation}

For optical simulation we use the software POV-Ray, a ray tracer for generating virtual optical images. The process consists of the following parts.

The DEM obtained from LiDAR is first converted to the USGS optional ASCII DEM format (.dem). Thereafter, it is converted to POV-Ray format (.POV) and imported into POV-Ray. In POV-Ray, the following components have to be defined: the position of an orthographic camera, a light source emitting parallel light and the object center.

The object center refers to the center of a box which contains the DEM exactly. That is:

$$
\vec{X}_{o c}=\left[L / 2, \quad B / 2, \quad\left(H_{\max }+H_{\min }\right) / 2\right]
$$

Where $L$ and $B$ are the length and width of the box and $H_{\text {max }}, H_{\text {min }}$ are the maximum and minimum of height in DEM.

The position of camera and light source can be chosen in these two values:

$$
\begin{gathered}
\vec{X}_{S A R}=\left[\begin{array}{lll}
L / 2, & B / 2-d, & H_{\text {mean }}+d / \tan (i)
\end{array}\right] \\
\vec{X}_{\text {opt }}=\left[\begin{array}{lll}
L / 2, & B / 2+d, \quad H_{\text {mean }}+d / \tan \left(90^{\circ}-i\right)
\end{array}\right]
\end{gathered}
$$

Where $d$ is any positive number, $H_{\text {mean }}=\left(H_{\max }+H_{\min }\right) / 2$, $i$ is the incidence angle of real SAR sensor.

Choosing different positions for camera and light source ( $\vec{X}_{\text {camera }}$ and $\vec{X}_{\text {light }}$ ) in POV-Ray, we can obtain different simulated optical images. The following three configurations for optical simulation are used. Their sketches are shown in Table I.

- $\quad$ Configuration 1: $\vec{X}_{\text {camera }}=\vec{X}_{\text {light }}=\vec{X}_{\text {opt }}$

- $\quad$ Configuration 2: $\vec{X}_{\text {camera }}=\vec{X}_{\text {opt }}, \vec{X}_{\text {light }}=\vec{X}_{S A R}$

- $\quad$ Configuration 3: $\vec{X}_{\text {camera }}=\vec{X}_{\text {light }}=\vec{X}_{S A R}$

The position of the sensor is defined in the south of the model. In respect of the azimuth angle of SAR, we rotate the model clockwise (270-azimuth).

In order to obtain the same pixel size as on the real SAR image, we should define the cover area of the camera and the size of the simulated image in the software as follows.

For deriving the image size, we project the four upper corners of the DEM-box onto the viewing direction of the camera. Via the difference between the maximum and minimum distance we get a value "up", which refers to the cover length in range direction. With the same principle we get the value "right" for the cover length of the camera in azimuth direction. Through a projection of this image in the horizontal plain, we get then an image with the same pixel size as the real SAR image. This projection can be simply done through a definition of the pixel size of the simulated image as $r_{\text {range }} \cos (i)$. Where, $r_{\text {range }}$ refers to the resolution in range direction for the real SAR image. The pixel size of the simulated image in azimuth direction is defined in the same way as for the real SAR image.

\section{SAR simulation}

For SAR simulation, RaySAR is used which has been developed at Remote Sensing Technology, Technische Universität München (TUM). It is based on POV-Ray [4] and provides output data for generating images in SAR geometry. The detailed process of SAR simulation is shown in [2].

\section{Geocoding of the simulated images}

For the simulation of optical images, all parameters are defined in POV-Ray. Each optical image has the same size and orientation (azimuth and slant range) as the simulated SAR 
image. Hence, the geocoding step for the simulated SAR images and optical images is the same.

The real TSX image is already orthorectified in east-north map. In order to compare it with the simulated images, the geocoding of the simulated image is necessary, what can be done based on the geocoded DEM.

Figure 2. shows the geometry for geocoding. The blue rectangle marks the area covered by the DEM. With POV-Ray and RaySAR we obtain simulated images within the area marked in black. With a clockwise rotation of (azimuth angle $90^{\circ}$ ), we get the green marked image, which is located in eastnorth position. With consideration of the projection in viewing direction of the camera, only the red marked area contains meaningful values. So we cut the border of the green marked image in both horizontal and vertical direction with range of $d 1=\|B \cos (A z) \sin (A z)\|$ and $d 2=\|L \cos (A z) \sin (A z)\|$, where $A z$ is the azimuth angle. The geocoding of this red image can then easily be conducted using the geocoded DEM. It has to be mentioned that there can be a shift between the red and blue rectangle as a result of different azimuth angle. We can calculate it from the projection of the upper left corner of DEM. The shift is $\left\|H \tan \left(90^{\circ}-i\right) \cos (A z)\right\|$ in east and $\left\|H \tan \left(90^{\circ}-i\right) \sin (A z)\right\|$ in south direction. The variable $H$ refers to the height difference in the DEM.

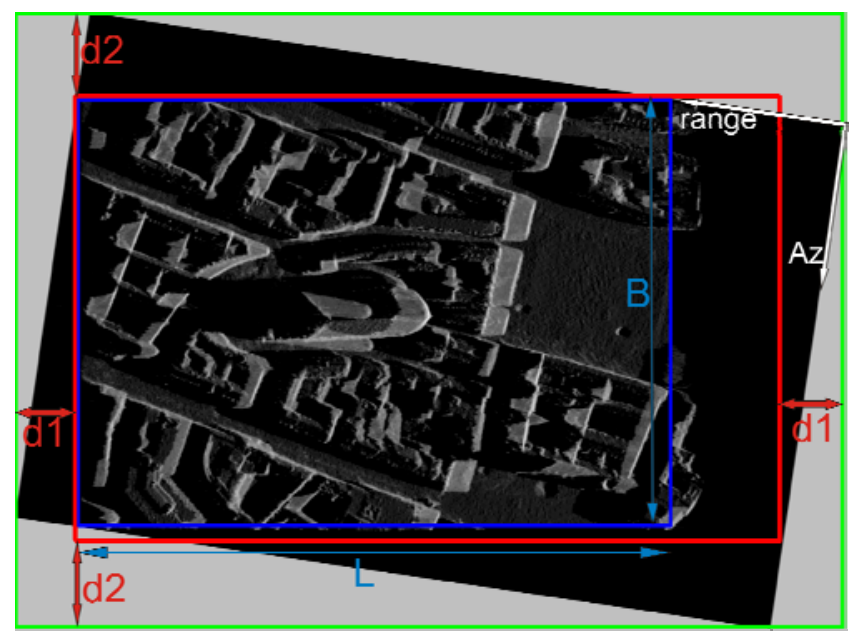

Figure 2. Geolocation of simulated images (blue rectangle: area of DEM; black: simulated image in azimuth and range direction; green: rotated simulated imge in east north direction; red: cropped image, final result)

\section{CASE STUDIES}

For the simulation we use a DEM of Munich city center with a horizontal and vertical resolution of 1 meter and 0.1 meter, respectively. The DEM is shown in Figure 3.

For this case study, we use the orthorectified TerraSAR-X (TSX) image (EEC product), having a pixel size of 0.5 meter, which has been captured on a descending orbit with an incidence angle of $50^{\circ}$.

Part A shows the simulation result of the optical simulation. Part B shows the result of the SAR simulation.

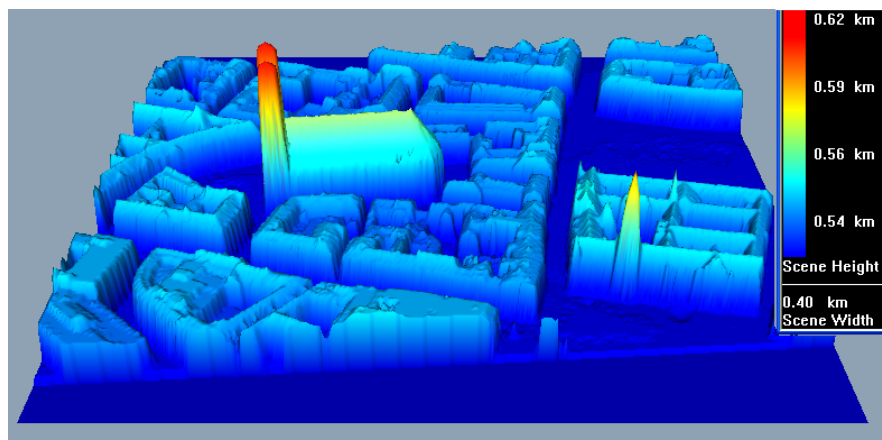

Figure 3. Digital elevation model of Munich center

\section{A. Result of optical simulation}

All geometrical parameters needed for POV-Ray have been calculated in consideration of the TSX data with the method explained in section II. In order to obtain a clear shape of the objects, all the model surfaces are characterized by low diffuse reflection for the optical simulation configuration 1 and by high diffuse scattering for configuration 2 . The geocoded simulated optical images of configuration 1 and 2 are presented in Figure 4. Compared to the TSX image, shown in Figure 5. , several similarities are clearly visible in the simulated optical images. Structures of high buildings (e.g. feature 1, top of Frauenkirche) and low buildings (e.g. feature 2) are found at the same geometrical position in the three images. Moreover, the shape of shadow areas is similar (e.g. feature 3). Vegetation (feature 5) is not represented in the DEM. Hence, it can not be seen in the simulated images. The simulated optical image of configuration 3 is not geocoded, because its geometry is not directly comparable with the TSX image.

Considering the analysis of the simulated optical images, the potential and limitations of the three acquisition configurations are summarized in TABLE I. For the visual interpretation of the SAR image, the configuration 2 is the most helpful, because the simulated image in this case present similarity not only on the geometry but also on the shadow.

TABLE I. DIFFERENT ACQUISITION CONFIGURATIONS

\begin{tabular}{|c|c|c|c|}
\hline $\begin{array}{l}\text { Acquisition } \\
\text { configurations }\end{array}$ & 1 & 2 & 3 \\
\hline sketch & $\begin{array}{ll}\quad \text { sun } & \text { SAR } \\
\text { Optical } & \text { Sensor } \\
\text { Sensor } & \text { S }\end{array}$ & sun $S A$ & $\begin{array}{l}\text { Optical sun } \\
\text { Sensor } \\
\text { SAR } \\
\text { Sensor }\end{array}$ \\
\hline advantage & $\begin{array}{l}\text { - same geometry } \\
\text { - contain the } \\
\text { invisible side of } \\
\text { SAR }\end{array}$ & $\begin{array}{l}\text { - same geometry } \\
\text { • same shadow }\end{array}$ & $\begin{array}{l}\text { - signal from same } \\
\text { object } \\
\text { - good to } \\
\text { understand } \\
\text { multiple } \\
\text { reflections }\end{array}$ \\
\hline disadvantage & different shadows & $\begin{array}{l}\text { no multiple } \\
\text { reflection signal }\end{array}$ & $\begin{array}{l}\text { different } \\
\text { geometries }\end{array}$ \\
\hline
\end{tabular}

If the incidence angle is smaller than the roof slope angle, not the whole roof is visible to the SAR and visual optical sensors and the shape of buildings will be different in the two images. This is a limitation of the interpretation of SAR images with simulated optical images. 

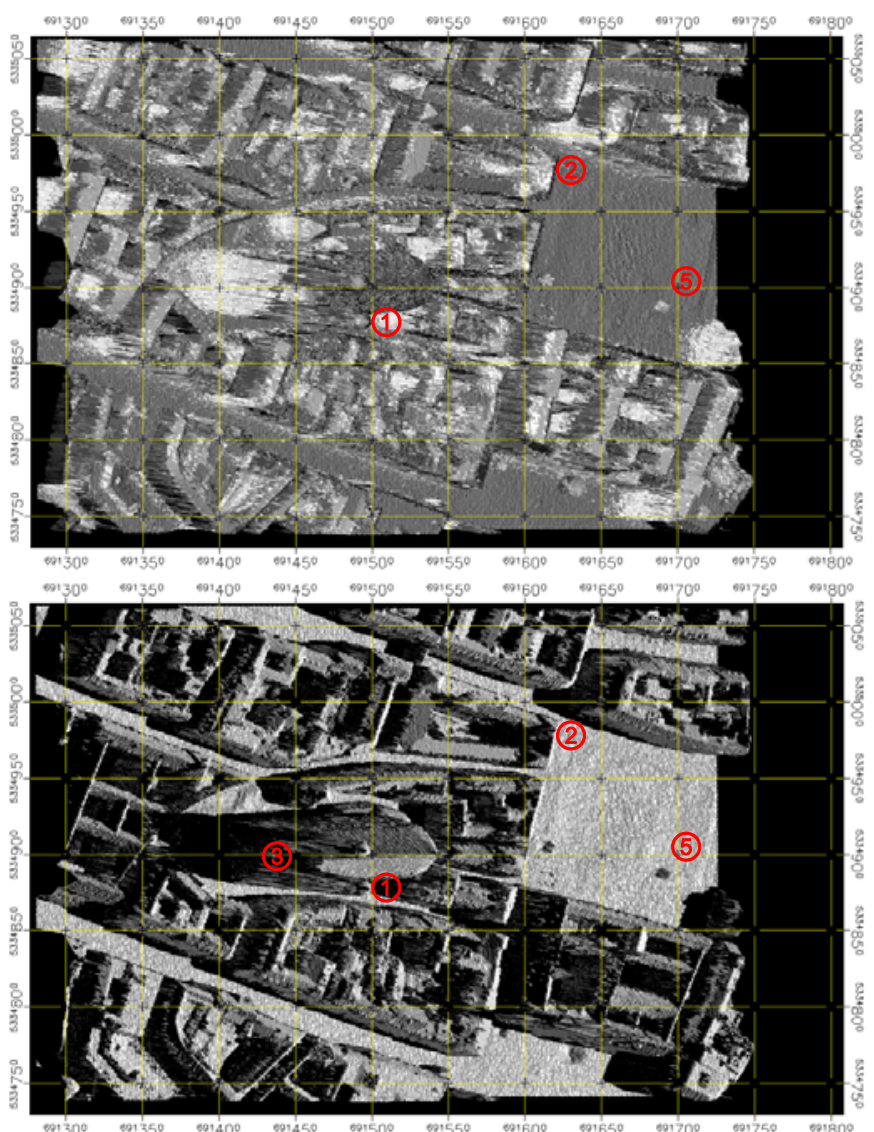

Figure 4. Geolocated simulated optical image of configuration 1 (upper image) and configuration 2 (lower image)

\section{B. Result of SAR simulation}

Based on the acquisition configuration 3, we get the geocoded simulated SAR image in RaySAR. The lower image in Figure 5. shows the simulated SAR image including reflection levels 1-3, which can be assigned to separate image layers [2]. Besides features 1-3, a linear signature, occurring at a wall corner due to the double reflection of radar signals (feature 4), shows similarity to the TSX image.

\section{CONCLUSION}

In this paper, a method for supporting the visual interpretation of SAR images with simulated optical and SAR images using LiDAR DEM has been presented. Since the location and shape of the objects are similarly represented in the simulated images, acquiring the semantic on a SAR image is eased. The simulated optical image can be used for direct and quick identification of objects in the SAR image. The simulated SAR image has a similar signal reflectivity as the TerraSAR-X image, and it can also separately present single or multiple scattering in the SAR images, which is very useful for building recognition and reconstruction. Both the simulated optical and SAR images are automatically geocoded and enable a direct comparison with the SAR images. Future work will concentrate on learning the semantic relationship between objects in optical and SAR images in order to improve, for instance, methods for change detection.
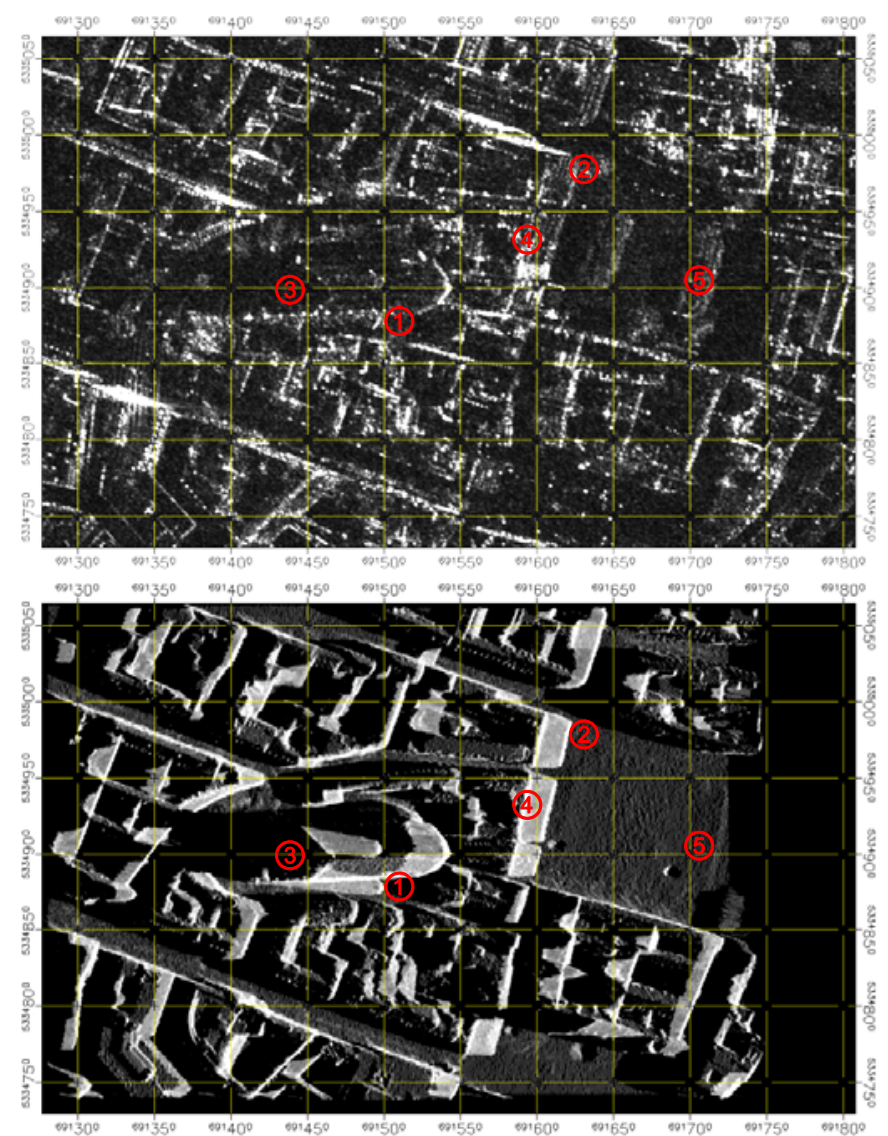

Figure 5. TerraSAR-X image (upper), simulated SAR image: all reflections (lower). Yellow grid lines with UTM coordinates are for better orientation between the images.

\section{REFERENCES}

[1] G. Palubinskas, P. Reinartz, R. Bamler, "Image acquisition geometry analysis for the fusion of optical and radar remote sensing data", International Journal of Image and Data Fusion, Vol. 1, Issue 3, September 2010, pp. $271-282$.

[2] S. Auer, S. Hinz, R. Bamler, "Ray-Tracing Simulation Techniques for Understanding High-Resolution SAR Images”, IEEE Transactions on Geoscience and Remoe Sensing, Vol. 48, No.3, March 2010. pp. 14451456.

[3] T. Balz, "SAR Simulation of Urban Areas: Techniques and Applications”, In book: Radar Remote Sensing of Urban Areas, Remote Sensing and Digital Image Processing, Springer Science, 2010, pp. 215231.

[4] Persistence of Vision Raytracer Propriety Limited. [last access:28.07.2009]. [Online]. Available: www.povray.org

[5] Thiele, A., Cadario, E., Schulz, K., Thonnessen, U., Soergel, U., "Building Recognition From Multi-Aspect High-Resolution InSAR Data in Urban Areas”, IEEE Transactions on Geoscience and Remote Sensing, 2007, 45, 3583-3593.

[6] Michaelsen, E., Soergel, U., Thoennessen, U., "Perceptual grouping for automatic detection of man-made structures in high-resolution SAR data”, Pattern Recogn. Lett., 2006, 27, 218-225.

[7] Auer, S., Balz, T., Becker, S., Bamler, R., “3D SAR Simulation of Urban Areas Based on Detailed Building Models”, Photogrammetric Engineering \& Remote Sensing, 2010, 76, 1373-1384. 
